\title{
NOUVELLE
}

\section{Foxa2, une nouvelle piste neuro-développementale pour le Parkinson}

Jacques Epelbaum

> Jusqu'à présent, les modèles animaux de la maladie de Parkinson étaient de deux types: soit des animaux chez lesquels une lésion chirurgicale ou neurotoxique des neurones dopaminergiques mésencéphaliques était créée, soit des modèles génétiques, fondés sur l'expression de l'un des quelques gènes mutés identifiés chez les rares patients avec une transmission génétique avérée ( $\alpha$-synucléine, Parkine, leucine-rich-repeat-kinase-2 [LRKK2]) [1, 2]. Cependant, aucun de ces modèles ne reproduisait la dégénérescence progressive, sélective et souvent asymétrique des neurones dopaminergiques de la voie nigro-striée $[3,4]^{1}$.

$\varepsilon$ n recherchant les facteurs de transcription qui spécifient le phénotype dopaminergique des neurones mésencéphaliques, à partir des cellules progénitrices du plancher du tube neural, Ron Mckay et al. ont mis en évidence le rôle déterminant d'un facteur de transcription de la famille forkhead, Foxa2 [5]. Les souris foxa $2^{-/-}$ne survivent pas audelà du jour embryonnaire $(\varepsilon) 10,5$. Les auteurs observent que, contrairement à ceux des souris témoins, les explants mésencéphaliques prélevés à $\varepsilon 8,5$ jours

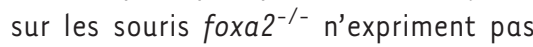
de tyrosine hydroxylase (TH), l'enzyme limitante de la voie de synthèse de la

${ }^{1}$ Voir la discussion en cours sur les modèles animaux des maladies neurodégénératives sur le Blog de $M / S$ : http:// forum.medecinesciences.org dopamine. En outre, la transfection de foxa2 dans des explants mésencéphaliques de $\varepsilon 10,5$ jours entraîne une augmentation massive du nombre de neurones exprimant la $\mathrm{TH}$.

Les auteurs suivirent ensuite une cohorte de souris hétérozygotes foxa2 $2^{+/-}$pour leurs performances motrices. À l'âge de 18 mois, un tiers des animaux com-

UMR 894 Inserm, Faculté de Médecine, Université Paris Descartes, 2ter, rue d'Alésia, 75014, Paris France. epelbaum@broca.inserm.fr

de Loewy, un marqueur pathognomonique de la maladie humaine, n'a pu être observé. Quoi qu'il en soit, les souris foxa2 $^{+/-}$sont bien le premier modèle animal qui reproduit l'atteinte progressive et asymétrique des neurones dopaminergiques nigro-striés.

Aux cliniciens de vérifier maintenant si certaines formes de Parkinson sont dues à un dysfonctionnement de Foxa2. Le fait que seul un tiers des souris foxa2 $2^{+/-}$est affecté offre la possibilité de rechercher des interactions génétiques, mais également environnementales, qui peuvent jouer un rôle dans la survie des neurones dopaminergiques in vivo. D’ores et déjà, l'implication de Foxa2 dans la spécification des neurones dopaminergiques est porteuse d'espoir pour les approches en thérapie cellulaire

mence à présenter une posture asymétrique avec une rigidité musculaire, progressant de la queue vers les hanches, puis le tronc des animaux. L'implication d'une perte dopaminergique fut vérifiée grâce au test de rotation induite par l'amphétamine.

Les animaux affectés présentent une perte asymétrique des neurones dopaminergiques de la substance noire, particulièrement de la sous-population exprimant la rétinaldéhyde déshydrogénase-1 (RALDHl). En revanche, une activation gliale est observée de manière symétrique, indépendamment de l'hémisphère touché, et aucun corps régénérative. $\diamond$

Foxa2, a new player in the

development of dopaminergic neurons

\section{RÉFÉRENCES}

1. Corti 0, Brice A. La maladie de Parkinson: que nous apprennent les gènes responsables des formes familiales? Med Sci (Paris) 2003; 19: 613-9.

2. Berthier A. Un peu de Rose dans la maladie de Parkinson. Med Sci (Paris) 2008 ; 24 : 118-9.

3. Langui D, Lachapelle F, Duyckaerts C. Modèles animaux des maladies neuro-dégénératives. Med Sci (Paris) $2007 ; 23: 180-6$.

4. Blog M/S. http://forum.medecinesciences.org/index2. lasso?id_sujet $=14$

5. Kittappa R, Chang WW, Awatramani RB, McKay RD. The foxa2 gene controls the birth and spontaneous degeneration of dopamine neurons in old age. PLoS Biol 2007 ; 5 : e325. 